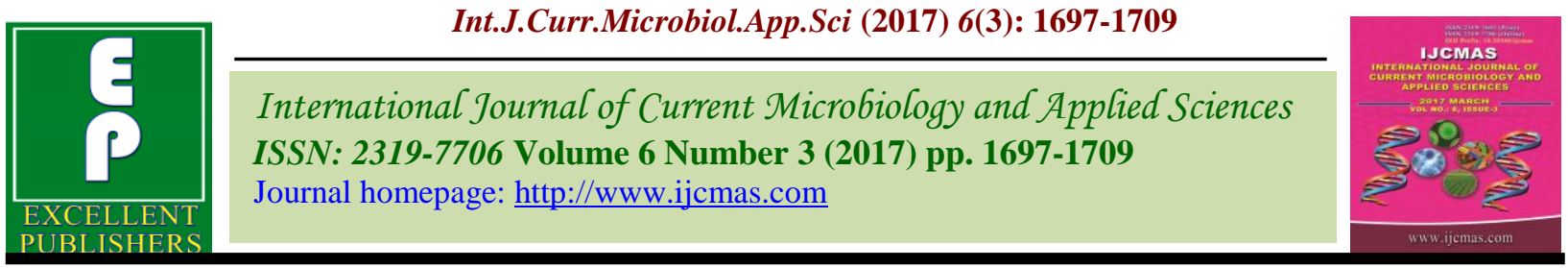

Original Research Article

https://doi.org/10.20546/ijcmas.2017.603.195

\title{
Virulence Factors of Acinetobacter baumannii Environmental Isolates and Their Inhibition by Natural Zeolite
}

\author{
Svjetlana Dekic $^{1}$, Jasna Hrenovic ${ }^{1}$, Blazenka Hunjak ${ }^{2}$, Snjezana Kazazic $^{3}$, \\ Darko Tibljas $^{1}$ and Tomislav Ivankovic ${ }^{1}$ \\ ${ }^{1}$ Faculty of Science, University of Zagreb, Zagreb, Croatia \\ ${ }^{2}$ Croatian Institute of Public Health, Zagreb, Croatia \\ ${ }^{3}$ Ruđer Boskovic Institute, Zagreb, Croatia \\ *Corresponding author
}

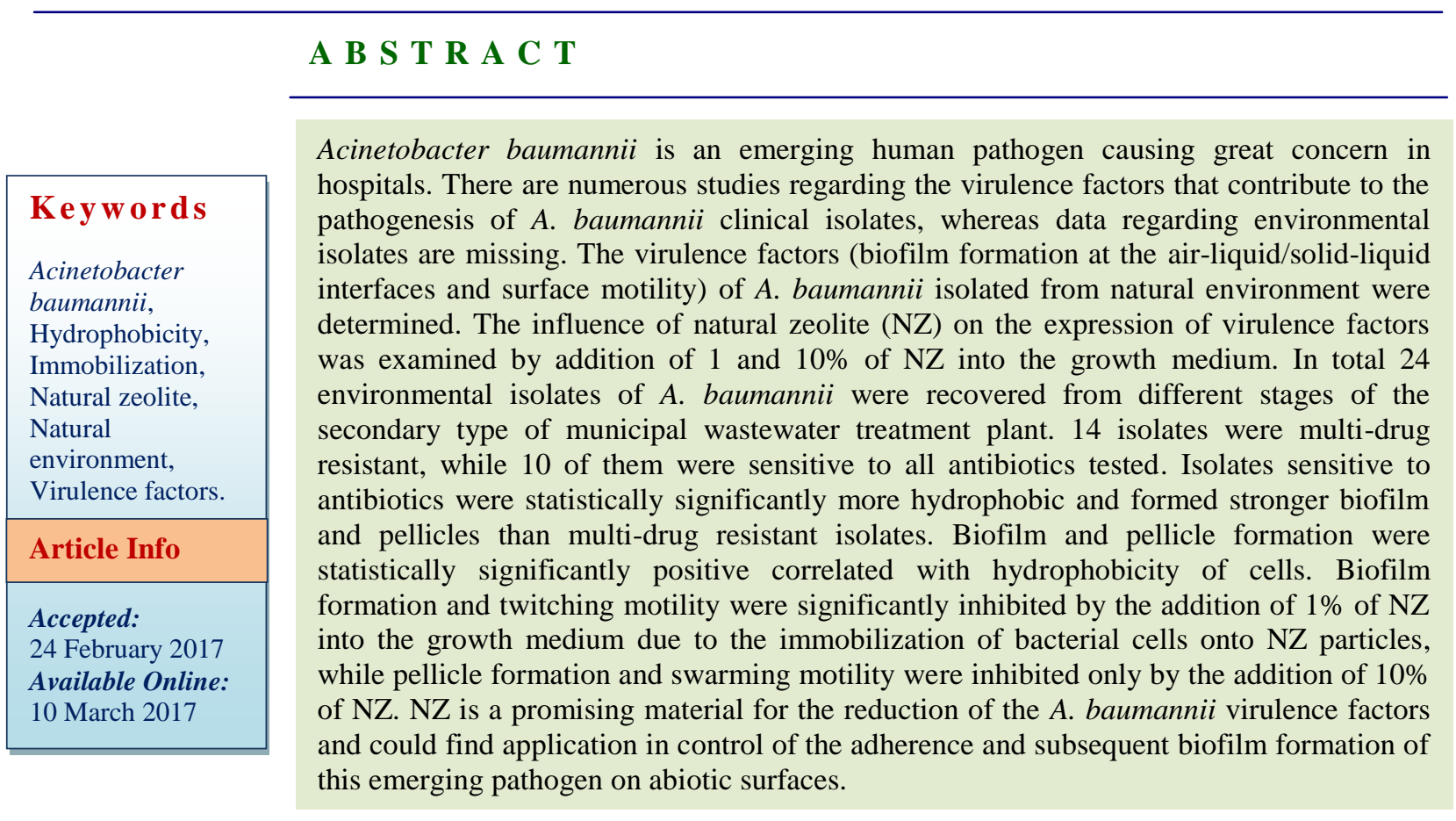

\section{Introduction}

Acinetobacter baumannii is an emerging human pathogen causing great concern in hospital environment over the last two decades. A. baumannii expresses the resistance to multiple antibiotics as well as disinfectants, and survives in adverse conditions, leading to long-term persistence in the hospital environment (Espinal et al., 2012; Towner, 2009). Additionally, virulence factors that influence the success of $A$. baumannii as a pathogen are its surface motility on solid/semisolid media and the ability to form biofilm on abiotic or biotic surfaces (McConnell et al., 2013).

Biofilm formation is considered as one of the main virulence factor in clinical isolates of $A$. baumannii. Biofilm is an assemblage of 
microbial cells enclosed in an extracellular matrix, which can be formed on wide variety of solid surfaces (Antunes et al., 2011). Biofilm provides protection from harsh environmental conditions, and therefore isolates which are strong biofilm producers survive longer in the environment (Espinal et al., 2012). Highly organized types of biofilm formed at the air-liquid interface are called pellicles (Nait Chabane et al., 2014). Pellicle formation is recognized as a feature of pathogenic strains of $A$. baumannii (Marti et al., 2011).

Bacteria in the form of pellicle might contribute to their persistence in the environment. A. baumannii is considered to be non-motile in liquid media due to the absence of flagella, but surface motility on solid/semisolid media was described. Two distinct forms of phenotypic surface motility of $A$. baumannii are recognized: twitching defined as surface translocation on solid surfaces and swarming defined as surface translocation on the semisolid media (Antunes et al., 2011; Eijkelkamp et al., 2011a). Twitching motility is considered as an important step in colonization and subsequent biofilm formation on medical devices, which is one of the main sources of hospital infections with $A$. baumannii. Although the bacterial motility is generally linked to increased virulence, there is no confirmation of the influence of motility on the virulence of A. baumannii.

In order to suppress the factors that contribute to the persistence and epidemicity of $A$. baumannii, recently attempts are made to elucidate underlying mechanisms and to suppress the expression of its virulence factors. Motility and biofilm formation of clinical strain of $A$. baumannii were found to be inhibited by blue light illumination and iron limitation (Eijkelkamp et al., 2011b; Mussi et al., 2010). However, blue light illumination and iron limiting conditions are difficult to achieve in the environment in order to be used for the suppression of virulence factors of A. baumannii. Among different types of natural zeolitizied tuff (NZ), those containing clinoptilolite are usually used in scientific studies as well as in industrial applications(Wong, 2009)on the base of its widespread occurrence in nature, price-easily accessibility and feasibility, cost effectiveness, large surface area, rigidity, surface functionality, thermal, mechanical and radiation stability. Particles of nontoxic NZ were shown to display a high affinity for the immobilization of different bacterial species including the Acinetobacter spp. (Hrenovic et al., 2005; Hrenovic et al., 2009; Hrenovic et al., 2011). Therefore, it was presumed that the addition of NZ into the growth medium will result in immobilization of $A$. baumannii cells onto NZ particles, thus hindering the expression of their virulence factors.

Due to its clinical relevance, A. baumannii is considered as an exclusive bacterium of the hospital environment. From 2010 onwards, continuous reports on the occurrence of $A$. baumannii outside hospital environment can be found. Multi-drug resistant (MDR) isolates of A. baumannii were found in hospital (Ferreira et al., 2011; Zhang et al., 2013)and municipal sewage (Goic-Barisic et al., 2017;Hrenovic et al., 2016), Seine River (Girlich et al., 2010), and in soil influenced by human solid waste (Hrenovic et al., 2014). However, to our knowledge there is no data on the phenotypic expression of the virulence factors that contribute to the pathogenesis in environmental isolates of $A$. baumannii. The aim of this study was to investigate the virulence factors of $A$. baumannii recovered from the natural environment, as well as the influence of NZ on the expression of biofilm and pellicle formation, swarming and twitching motility. 
Materials and Methods

\section{Isolation and characterization of $A$. baumannii}

The samples of influent and effluent wastewater, fresh activated sludge, and sludge passed through the anaerobic mesophilic digestion were collected between September 2015 and March 2016 at the secondary type municipal wastewater treatment plant of the City of Zagreb, Croatia. The isolation of $A$. baumannii was performed according to Hrenović et al.(2016)at $42^{\circ} \mathrm{C} / 48 \mathrm{~h}$ on CHROMagar Acinetobacter (CHROMagar) supplemented with $15 \mathrm{mg} / \mathrm{L}$ of cefsulodin sodium salt hydrate(Sigma-Aldrich). Identification of isolates was performed by routine bacteriological techniques, Vitek 2 system (BioMerieux), and MALDI-TOF MS (software version 3.0, Microflex LT, Bruker Daltonics) on cell extracts (Sousa et al., 2014). Susceptibility testing was done by Vitek 2 system and confirmed by gradient dilution E-test for colistin. Minimum inhibitory concentrations (MIC) were interpreted according to European Committee on Antimicrobial Susceptibility Testing (2016) criteria for all antibiotics with defined breakpoints for Acinetobacter spp., while for penicillins/ $\beta$-lactamase inhibitors and minocycline Clinical and Laboratory Standards Institute (2013) breakpoints were used.

\section{Bacterial hydrophobicity}

Hydrophobicity of bacteria was measured via the bacterial adhesion to hydrocarbon (BATH) assay according to Rosenberg et al., (1980)with slight modifications. The assay is based on the affinity of bacterial cells for an organic hydrocarbon such as hexadecane. More hydrophobic bacteria will migrate from aqueous suspension to the hexadecane layer, resulting in reduction of bacterial concentration in the water phase. Overnight bacterial culture was suspended in $5 \mathrm{~mL}$ of phosphate-buffered saline (PBS), $0.5 \mathrm{~mL}$ of $\mathrm{n}$ hexadecane was added to the suspension, shaken for $10 \mathrm{~min}$ and left to stand for $2 \mathrm{~min}$. The reduction in bacterial concentration was measured spectrophotometrically (DR/2500 Hach spectrophotometer) at absorbance of $410 \mathrm{~nm}\left(\mathrm{OD}_{410}\right)$ both before and after the addition of $\mathrm{n}$-hexadecane.

\section{Natural zeolitizied tuff}

The NZ was obtained from quarries located at Donje Jesenje, Croatia. The main constituent of NZ is clinoptilolite (50-55\%). Other major constituents (10-15\% each) are celadonite, plagioclase feldspars and opal-CT, while analcime and quartz are present in traces (Hrenovic et al., 2011). The NZ was crushed, sieved, and the size fraction less than $0.122 \mathrm{~mm}$ was used. Prior to its usage in experiments, dry NZ was sterilized by autoclaving.

\section{Biofilm formation}

The ability to form biofilm in vitro was tested via the crystal violet assay (Kaliterna et al., 2015). Overnight bacterial culture was diluted in nutrient broth (Biolife) to an absorbance of 0.1 at $600 \mathrm{~nm}\left(\mathrm{OD}_{600}\right)$. The suspension was distributed into the polypropylene tubes and then incubated at $37^{\circ} \mathrm{C} / 48 \mathrm{~h}$ without shaking. After incubation, the planktonic bacteria were removed and the tubes were gently washed with PBS. Biofilm was stained with $0.5 \%$ (w/v) crystal violet at $37^{\circ} \mathrm{C} / 20 \mathrm{~min}$. After solubilisation with $96 \%$ ethanol at $37^{\circ} \mathrm{C} / 20$ min, biofilm was quantified at $550 \mathrm{~nm}$ $\left(\mathrm{OD}_{550}\right)$. The estimated criteria used to interpret the biofilm formation were: $\mathrm{OD}_{550}$ value beneath 0.3 poor biofilm formers; value between 0.3 and 1 intermediate biofilm formers; value above 1.0 strong biofilm formers. The procedure was repeated with the 
addition of $1 \% \mathrm{NZ}$ into the bacterial suspension for all isolates, while $10 \%$ of NZ was added into the suspension of selected intermediate and strong biofilm formers.

\section{Pellicle formation}

Pellicle formation assay was performed according to the protocol described in Nait Chabane et al., (2014). Overnight bacterial culture with the initial concentration of 0.01 at an $\mathrm{OD}_{600}$ was divided into the polystyrene tubes with $2 \mathrm{~mL}$ of Mueller Hinton Broth (Biolife) and incubated at $25^{\circ} \mathrm{C} / 72 \mathrm{~h}$. Pellicle formation was identified visually and its cohesion was examined by inverting the tubes. Cohesion of pellicles was divided into three categories: no pellicle formation (0); poor pellicle formation (1); strong pellicle formation (2). The procedure was repeated with the addition of 1 and 10\% of NZ for isolates which formed poor and strong pellicles.

\section{Swarming and twitching surface motility}

Swarming and twitching surface motility was assessed according to Antunes et al. (2011). For surface motility study Luria-Bertani medium with $0.5 \%$ agarose was used, into which0, 1 and $10 \%$ of NZ was added. Overnight bacterial cultures were suspended in $1 \mathrm{~mL}$ PBS and inoculated with a pipette tip to the bottom of the polystyrene Petri dish, tightly closed with parafilm and incubated in humid atmosphere at $37^{\circ} \mathrm{C} / 24 \mathrm{~h}$. Swarming motility was observed at the air-agarose interface by direct measuring of the longest diameter of motility. Twitching motility was determined after the removal of the agarose layer, staining the Petri dish with $0.5 \%$ crystal violet for $10 \mathrm{~min}$ and measuring the longest diameter of motility. Isolates were grouped into categories based on the average values of motility: < $25 \mathrm{~mm}$ poor; $25-50 \mathrm{~mm}$ intermediate; $>50 \mathrm{~mm}$ highly motile isolates.
To confirm the immobilization of bacteria onto NZ, particles of NZ were taken at the end of experiments on motility and biofilm formation. Particles were stained with carbol fuxin dye and examined under optical microscope (Olympus CX21) at magnification of $1000 x$.

\section{Data analyses}

All experiments were performed in biological and technical duplicate with mean values presented. Percentages of reduction were calculated for each isolate with addition of $\mathrm{NZ}$ as compared to the same isolate without $\mathrm{NZ}$ addition. Statistical analyses were carried out using Statistica software 12 (StatSoft, Inc.). The comparisons between samples were done by using the ordinary Student's $t$-test for independent variables. The correlation between variables was estimated by Pearson linear correlation analysis. Statistical decisions were made at a significance level of $\mathrm{p}<0.05$.

\section{Results and Discussion}

\section{Characterization of $A$. baumannii isolates}

In total24 environmental isolates of $A$. baumannii have been isolated from 4 different stages of municipal wastewater treatment plant (6 per each stage of treatment): influent wastewater, effluent wastewater, fresh activated sludge and digested sludge. The list of recovered isolates, their origin, date of isolation, and MALDI-TOF MS score values are given in table 1 .

The antibiotic resistance profile of isolates is shown in Table 2. From each stage of the wastewater treatment plant, isolates sensitive to all 12tested antibiotics (10 isolates), as well as MDR isolates (14 isolates) were chosen for study. MDR isolates shared the resistance to carbapenems and fluoroquinolones, but 
sensitivity to colistin. The pan drug-resistant isolate EF7 has already been described in Goic-Barisic et al. (2017).

Significant hydrophobicity, estimated as migration of cells to hydrocarbon of $46 \%$ and higher, was observed for $2 / 6$ isolates from influent wastewater, 1/6 isolates from effluent wastewater, 3/6 isolates from fresh activated sludge, and $3 / 6$ isolates from digested sludge (Table 2). In total $9 / 24$ isolates from wastewater treatment plant were hydrophobic. 7/9 hydrophobic isolates were sensitive to tested antibiotics, while 2 remaining hydrophobic isolates which were MDR showed the lower level of hydrophobicity than sensitive isolates. Isolates sensitive to all tested antibiotics were statistically significantly more hydrophobic than MDR isolates $(\mathrm{p}=0.000)$.

\section{Biofilm formation}

The results of biofilm formation of isolates are presented in Fig. 1. Great proportion $(14 / 24)$ of isolates were intermediate biofilm formers $\left(\mathrm{OD}_{550} 0.3-1.0\right)$, whereas only $3 / 24$ (IN41, D12, D13) formed poor biofilm. Among 7strong biofilm formers, the isolate IN58 stands out with an $\mathrm{OD}_{550}$ value of 2.5.Isolates sensitive to antibiotics formed stronger biofilm than MDR isolates $(\mathrm{p}=0.005)$.

Biofilm formation showed statistically significant positive correlation with hydrophobicity of cells $(\mathrm{r}=0.425, \mathrm{p}=0.003$, Table 3).With the addition of $1 \%$ of NZ biofilm formation dropped significantly ( $\mathrm{p}=0.003$, Fig. 1). With the addition of $10 \%$ of NZ to selected isolates, biofilm formation dropped significantly even further $(\mathrm{p}=0.002)$.Average percentage of inhibition for isolates were $39 \pm 21 \%$ and $76 \pm 21 \%$ with the addition of 1 and $10 \%$ of $\mathrm{NZ}$, respectively.

\section{Pellicle formation}

Majority (19/24) of isolates formed poor pellicles, while only isolate IN41 formed no pellicle. Isolates EF11, S9, D17 and especially IN58 formed strong pellicles (Table 2). Among 4 isolates which formed strong pellicles, 3 were hydrophobic and sensitive to antibiotics, while this does not stand only for isolate D17. Pellicle formation showed statistically significant positive correlation with cell hydrophobicity ( $\mathrm{r}=0.433$, $\mathrm{p}=0.002$ ), as well as with the biofilm formation $(r=0.682, p=0.000$, Table 3$)$. The addition of $1 \%$ of $\mathrm{NZ}$ did not influence the pellicle formation (data not shown).However, $10 \%$ of NZ decreased the consistency of pellicles from strong to intermediate consistency.

\section{Swarming and twitching surface motility}

The results presented in Figs. 2 and 3indicate that all examined environmental isolates of $A$. baumannii expressed the surface motility by swarming or twitching.10/24 isolates showed poor swarming, whereas $8 / 24$ and $6 / 24$ isolates showed intermediate and high swarming, respectively. Only $3 / 24$ isolates showed poor twitching, whereas 11/24 and $10 / 24$ isolates showed intermediate and high twitching, respectively. No connection of surface motility and sensitivity or MDR of isolates to antibiotics could be established. Swarming and twitching motility were not mutually linked parameters ( $\mathrm{r}=-0.018$, $\mathrm{p}=0.904$ ) and showed no correlation with cell hydrophobicity, biofilm or pellicle formation (Table 3).

The addition of $1 \%$ of NZ significantly increased the swarming motility of isolates $(47 \pm 21 \%$ increase), while the addition of $10 \%$ of NZ had no statistically significant influence on swarming $(18 \pm 51 \%$ reduction, Fig. 2). Contrary to swarming, twitching 
motility was significantly reduced by $1 \%$ of NZ (48 $\pm 19 \%$ reduction, $p=0.000)$ and $10 \%$ of NZ reduced twitching even further $(52 \pm 20 \%$ reduction, $p=0.001$, Fig. 3 ), but there was no statistically significant difference between addition of 1 and $10 \%$ of NZ. In order to elucidate the mechanism of significant reduction of biofilm formation and twitching motility by the addition of NZ, the particles of $\mathrm{NZ}$ were examined at the end of experiments for the immobilization of $A$. baumannii. Microscopic examination confirmed the immobilization of cells of A. baumannii onto NZ particles in high extent (Fig. 4).

Table1. Origin, date of isolation, MALDI-TOF MS score values, hydrophobicity values, and pellicle formation of $A$. baumannii isolates. Isolates with hydrophobicity higher than $46 \%$ are considered hydrophobic. Cohesionof pellicles was divided into three categories: no pellicle formation (0), poor pellicle formation (1); strong pellicle formation (2).

\begin{tabular}{|c|c|c|c|c|c|}
\hline Isolate & Origin & $\begin{array}{c}\text { Date of } \\
\text { isolation }\end{array}$ & $\begin{array}{l}\text { MALDI- } \\
\text { TOF score }\end{array}$ & $\begin{array}{l}\text { Hydrophobicity } \\
\left(\% \mathrm{OD}_{410}\right)\end{array}$ & $\begin{array}{c}\text { Pellicle } \\
\text { formation }\end{array}$ \\
\hline IN31 & Influent & 23.9 .2015 & 2.119 & 97.15 & 1 \\
\hline IN34 & Influent & 23.9 .2015 & 2.066 & 0.66 & 1 \\
\hline IN36 & Influent & 23.9 .2015 & 2.184 & 1.97 & 1 \\
\hline IN41 & Influent & 4.11 .2015 & 2.068 & 0.00 & 0 \\
\hline IN47 & Influent & 18.11 .2015 & 2.198 & 0.00 & 1 \\
\hline IN58 & Influent & 26.1 .2016 & 2.205 & 92.76 & 2 \\
\hline EF7 & Effluent & 9.9 .2015 & 2.150 & 0.00 & 1 \\
\hline EF8 & Effluent & 23.9 .2015 & 2.180 & 0.00 & 1 \\
\hline EF11 & Effluent & 18.11 .2015 & 2.173 & 80.00 & 2 \\
\hline EF13 & Effluent & 2.12 .2015 & 2.074 & 0.00 & 1 \\
\hline EF22 & Effluent & 26.1 .2016 & 2.149 & 0.00 & 1 \\
\hline EF23 & Effluent & 26.1.2016 & 2.189 & 0.00 & 1 \\
\hline S5 & Fresh sludge & 23.9 .2015 & 2.178 & 2.54 & 1 \\
\hline S6 & Fresh sludge & 4.11 .2015 & 2.247 & 78.04 & 1 \\
\hline S9 & Fresh sludge & 26.1 .2016 & 2.063 & 80.00 & 2 \\
\hline S10 & Fresh sludge & 10.2 .2016 & 2.025 & 1.69 & 1 \\
\hline S11 & Fresh sludge & 10.2 .2016 & 2.079 & 0.00 & 1 \\
\hline S15 & Fresh sludge & 23.3.2016 & 2.000 & 78.84 & 1 \\
\hline D10 & Digested sludge & 23.9 .2015 & 2.248 & 0.00 & 1 \\
\hline D11 & Digested sludge & 14.10 .2015 & 2.103 & 46.36 & 1 \\
\hline D12 & Digested sludge & 14.10 .2015 & 2.037 & 48.63 & 1 \\
\hline D13 & Digested sludge & 18.11 .2015 & 2.048 & 0.00 & 1 \\
\hline D16 & Digested sludge & 26.1 .2016 & 2.081 & 67.36 & 1 \\
\hline D17 & Digested sludge & 10.2 .2016 & 2.253 & 1.40 & 2 \\
\hline
\end{tabular}


Table.2 MIC values of tested antibiotics against isolates of $A$. baumannii.

\begin{tabular}{|c|c|c|c|c|c|c|c|c|c|c|c|c|}
\hline \multirow[b]{2}{*}{ Isolate } & \multicolumn{12}{|c|}{ MIC values of antibiotics (mg/L) } \\
\hline & MEM & IMI & CIP & LVX & TOB & GEN & AMK & MIN & SAM & TIM & SXT & CST \\
\hline IN31 & $<0.25$ & $<0.25$ & $<0.25$ & $<0.12$ & $<1$ & $<1$ & $<2$ & $<1$ & $<2$ & $<8$ & $<20$ & $\leq 0.5$ \\
\hline IN34 & $>16^{\mathrm{R}}$ & $>16^{\mathrm{R}}$ & $>4^{\mathrm{R}}$ & $8^{\mathrm{R}}$ & $>16^{\mathrm{R}}$ & $>16^{\mathrm{R}}$ & $>64^{\mathrm{R}}$ & $>16^{\mathrm{R}}$ & $>32^{\mathrm{R}}$ & $>128^{\mathrm{R}}$ & $<20$ & $\leq 0.5$ \\
\hline IN36 & $<0.25$ & $<0.25$ & $<0.25$ & $<0.12$ & $<1$ & $<1$ & $<2$ & $<1$ & $<2$ & $<8$ & $<20$ & $\leq 0.5$ \\
\hline IN41 & $\geq 16^{\mathrm{R}}$ & $\geq 16^{\mathrm{R}}$ & $\geq 4^{\mathrm{R}}$ & $\geq 8^{\mathrm{R}}$ & $\leq 1$ & $\leq 1$ & $\geq 64^{\mathrm{R}}$ & $8^{I}$ & $\geq 32^{\mathrm{R}}$ & $\geq 128^{\mathrm{R}}$ & $\geq 320^{\mathrm{R}}$ & $\leq 0.5$ \\
\hline IN47 & $\geq 16^{\mathrm{R}}$ & $\geq 16^{\mathrm{R}}$ & $\geq 4^{\mathrm{R}}$ & $\geq 8^{\mathrm{R}}$ & $\geq 16^{\mathrm{R}}$ & $8^{R}$ & $\geq 64^{\mathrm{R}}$ & $8^{I}$ & $16^{\mathrm{I}}$ & $\geq 128^{\mathrm{R}}$ & $\geq 320^{\mathrm{R}}$ & $\leq 0.5$ \\
\hline IN58 & $\leq 0.25$ & $\leq 0.25$ & $\leq 0.25$ & $\leq 0.12$ & $\leq 1$ & $\leq 1$ & $\leq 2$ & $\leq 1$ & $\leq 2$ & $\leq 8$ & $\leq 20$ & $\leq 0.5$ \\
\hline EF7 & $>16^{\mathrm{R}}$ & $>16^{\mathrm{R}}$ & $>4^{\mathrm{R}}$ & $>8^{\mathrm{R}}$ & $>16^{\mathrm{R}}$ & $>16^{\mathrm{R}}$ & $>64^{\mathrm{R}}$ & $8^{I}$ & $>32^{\mathrm{R}}$ & $>128^{\mathrm{R}}$ & $>320^{\mathrm{R}}$ & $16^{\mathrm{R}}$ \\
\hline EF8 & $\geq 16^{\mathrm{R}}$ & $\geq 16^{\mathrm{R}}$ & $\geq 4^{\mathrm{R}}$ & $\geq 8^{\mathrm{R}}$ & $\geq 16^{\mathrm{R}}$ & $\geq 16^{\mathrm{R}}$ & 8 & $\geq 16^{\mathrm{R}}$ & $\geq 32^{\mathrm{R}}$ & $\geq 128^{\mathrm{R}}$ & $\leq 20$ & $\leq 0.5$ \\
\hline EF11 & $\leq 0.25$ & $\leq 0.25$ & $\leq 0.25$ & $\leq 0.12$ & $\leq 1$ & $\leq 1$ & $\leq 2$ & $\leq 1$ & $\leq 2$ & $\leq 8$ & $\leq 20$ & $\leq 0.5$ \\
\hline EF13 & $\geq 16^{\mathrm{R}}$ & $\geq 16^{\mathrm{R}}$ & $\geq 4^{\mathrm{R}}$ & $\geq 8^{\mathrm{R}}$ & $\geq 16^{\mathrm{R}}$ & $\geq 16^{\mathrm{R}}$ & $\geq 64^{\mathrm{R}}$ & $\geq 16^{\mathrm{R}}$ & $\geq 32^{\mathrm{R}}$ & $\geq 128^{\mathrm{R}}$ & $\leq 20$ & $\leq 0.5$ \\
\hline EF22 & $\geq 16^{\mathrm{R}}$ & $\geq 16^{\mathrm{R}}$ & $\geq 4^{\mathrm{R}}$ & $4^{R}$ & $8^{\mathrm{R}}$ & 2 & 8 & 2 & $16^{\mathrm{I}}$ & $\geq 128^{\mathrm{R}}$ & $\geq 320^{R}$ & $\leq 0.5$ \\
\hline EF23 & $\geq 16^{\mathrm{R}}$ & $\geq 16^{\mathrm{R}}$ & $\geq 4^{\mathrm{R}}$ & $4^{R}$ & $\geq 16^{\mathrm{R}}$ & 4 & $16^{1}$ & 4 & $16^{\mathrm{I}}$ & $\geq 128^{\mathrm{R}}$ & $\geq 320^{\mathrm{R}}$ & $\leq 0.5$ \\
\hline S5 & $>16^{\mathrm{R}}$ & $>16^{\mathrm{R}}$ & $>4^{\mathrm{R}}$ & $>8^{R}$ & $>16^{\mathrm{R}}$ & $>16^{\mathrm{R}}$ & $>64^{\mathrm{R}}$ & $>16^{\mathrm{R}}$ & $>32^{\mathrm{R}}$ & $>128^{\mathrm{R}}$ & $<20$ & $\leq 0.5$ \\
\hline S6 & $\leq 0.25$ & $\leq 0.25$ & $\leq 0.25$ & $\leq 0.12$ & $\leq 1$ & $\leq 1$ & $\leq 2$ & $\leq 1$ & $\leq 2$ & $\leq 8$ & $\leq 20$ & $\leq 0.5$ \\
\hline S9 & $\leq 0.25$ & $\leq 0.25$ & $\leq 0.25$ & $\leq 0.12$ & $\leq 1$ & $\leq 1$ & $\leq 2$ & $\leq 1$ & $\leq 2$ & $\leq 8$ & $\leq 20$ & $\leq 0.5$ \\
\hline S10 & $\geq 16^{\mathrm{R}}$ & $\geq 16^{\mathrm{R}}$ & $\geq 4^{\mathrm{R}}$ & $\geq 8^{\mathrm{R}}$ & $\geq 16^{\mathrm{R}}$ & $\geq 16^{\mathrm{R}}$ & $\geq 64^{\mathrm{R}}$ & $8^{I}$ & $16^{\mathrm{I}}$ & $\geq$ & $\geq 320^{\mathrm{R}}$ & $\leq 0.5$ \\
\hline S11 & $\geq 16^{\mathrm{R}}$ & $\geq 16^{\mathrm{R}}$ & $\geq 4^{\mathrm{R}}$ & $\geq 8^{\mathrm{R}}$ & 4 & $\geq 16^{\mathrm{R}}$ & $\geq 64^{\mathrm{R}}$ & $8^{I}$ & $16^{\mathrm{I}}$ & $\geq 128^{\mathrm{R}}$ & $\geq 320^{\mathrm{R}}$ & $\leq 0.5$ \\
\hline S15 & $\leq 0.25$ & $\leq 0.25$ & $\leq 0.25$ & $\leq 0.12$ & $\leq 1$ & $\leq 1$ & $\leq 2$ & $\leq 1$ & $\leq 2$ & $\leq 8$ & $\leq 20$ & $\leq 0.5$ \\
\hline D10 & 0.5 & $<0.25$ & $<0.25$ & $<0.12$ & $<1$ & $<1$ & $<2$ & $<1$ & $<2$ & $<8$ & $<20$ & $\leq 0.5$ \\
\hline D11 & $\geq 16^{\mathrm{R}}$ & $\geq 16^{\mathrm{R}}$ & $\geq 4^{\mathrm{R}}$ & $4^{\mathrm{R}}$ & $\leq 1$ & $\leq 1$ & $32^{\mathrm{R}}$ & $\leq 1$ & $16^{\mathrm{I}}$ & $\geq 128^{\mathrm{R}}$ & $\leq 20$ & $\leq 0.5$ \\
\hline D12 & $\geq 16^{\mathrm{R}}$ & $\geq 16^{\mathrm{R}}$ & $\geq 4^{\mathrm{R}}$ & $4^{\mathrm{R}}$ & $\leq 1$ & $\leq 1$ & $16^{\mathrm{I}}$ & $\leq 1$ & $16^{\mathrm{I}}$ & $\geq 128^{\mathrm{R}}$ & $\leq 20$ & $\leq 0.5$ \\
\hline D13 & $\leq 0.25$ & $\leq 0.25$ & $\leq 0.25$ & $\leq 0.12$ & $\leq 1$ & $\leq 1$ & $\leq 2$ & $\leq 1$ & $\leq 2$ & $\leq 8$ & $\leq 20$ & $\leq 0.5$ \\
\hline D16 & $\leq 0.25$ & $\leq 0.25$ & $\leq 0.25$ & $\leq 0.12$ & $\leq 1$ & $\leq 1$ & $\leq 2$ & $\leq 1$ & $\leq 2$ & $\leq 8$ & $\leq 20$ & $\leq 0.5$ \\
\hline D17 & $\geq 16^{\mathrm{R}}$ & $\geq 16^{\mathrm{R}}$ & $\geq 4^{\mathrm{R}}$ & $\geq 8^{\mathrm{R}}$ & $\geq 16^{\mathrm{R}}$ & $\geq 16^{\mathrm{R}}$ & $\geq 64^{\mathrm{R}}$ & $8^{I}$ & $16^{\mathrm{I}}$ & $\geq 128^{\mathrm{R}}$ & $\geq 320^{\mathrm{R}}$ & $\leq 0.5$ \\
\hline
\end{tabular}

${ }^{a}$ carbapenems (MEM-meropenem, IMI-imipenem), fluoroquinolones (CIP-ciprofloxacin, LVXlevofloxacin), aminoglycosides (TOB-tobramycin, GEN-gentamicin, AMK-amikacin), tetracyclines (MIN-minocycline), penicillins/ $\beta$-lactamase inhibitors (SAMampicillin/sulbactam,TIM-ticarcillin/clavulanic acid), SXT- trimethoprim/sulfamethoxazole, CST-colistin. ${ }^{\mathrm{R}}$ - resistant, ${ }^{\mathrm{I}}$ - intermediate according to EUCAST or CLSI criteria.IN - influent wastewater, EF -effluent wastewater, S - fresh sludge, D - digested sludge isolates. 
Table.3 Summary of the correlation parameters for the expression of virulence factors of $A$. baumannii isolates

\begin{tabular}{|l|c|c|c|c|c|}
\hline & Hydrophobicity & Biofilm & Pellicle & Swarming & Twitching \\
\hline Hydrophobicity & 1.000 & $\boldsymbol{r}=\mathbf{0 . 4 2 5}$, & $\boldsymbol{r}=\mathbf{0 . 4 3 3 ,}$ & $\mathrm{r}=-0.142$, & $\mathrm{r}=0.249$, \\
& & $\boldsymbol{p}=\mathbf{0 . 0 0 3}$ & $\boldsymbol{p = 0 . 0 0 2}$ & $\mathrm{p}=0.335$ & $\mathrm{p}=0.088$ \\
\hline Biofilm & 1.000 & $\boldsymbol{r}=\mathbf{0 . 6 8 2}$, & $\mathrm{r}=-0.123$, & $\mathrm{r}=-0.049$, \\
& & & $\boldsymbol{p}=\mathbf{0 . 0 0 0}$ & $\mathrm{p}=0.405$ & $\mathrm{p}=0.740$ \\
\hline Pellicle & & 1.000 & $\mathrm{r}=0.096$, & $\mathrm{r}=-0.028$, \\
& & & $\mathrm{p}=0.518$ & $\mathrm{p}=0.851$ \\
\hline Swarming & & & & 1.000 & $\mathrm{r}=-0.018$, \\
& & & & & $\mathrm{p}=0.904$ \\
\hline Twitching & & & & & 1.000 \\
\hline
\end{tabular}

Fig.1 Biofilm formation $\left(\mathrm{OD}_{550}\right)$ without natural zeolite $(0 \% \mathrm{NZ})$, with $1 \%$ of $\mathrm{NZ}$, and for selected isolates with $10 \%$ of NZ. Lines represent boundaries: $\mathrm{OD}_{550}<0.3$ poor, $\mathrm{OD}_{550} 0.3-1.0$ intermediate, $\mathrm{OD}_{550}>1.0$ strong biofilm formation

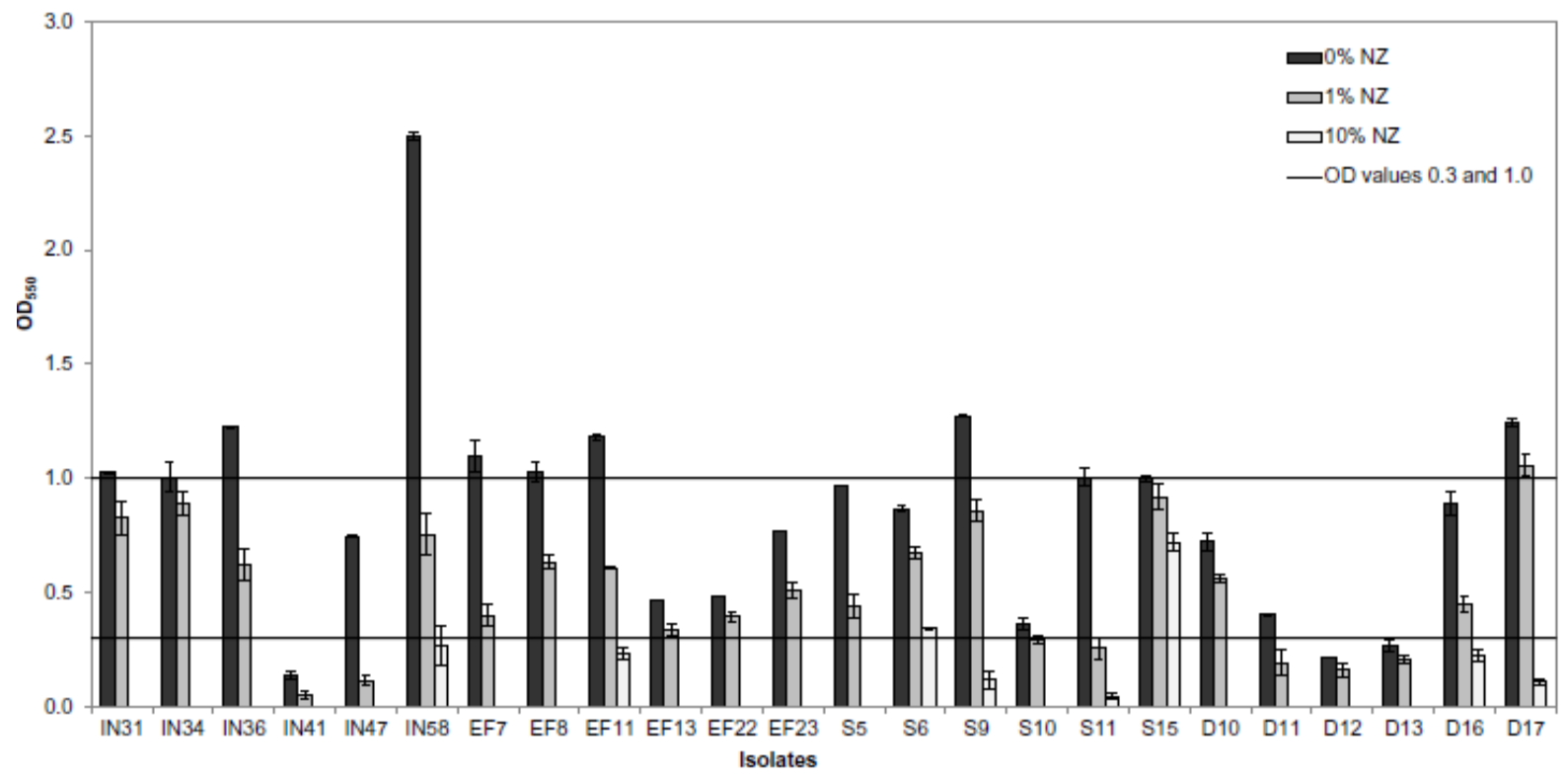


Fig.2 Swarming motility without natural zeolite ( $0 \%$ NZ), with $1 \%$ of NZ, and for selected isolates with $10 \%$ of NZ. Lines represent boundaries: <25mmpoor, $25-50 \mathrm{~mm}$ intermediate, $>50 \mathrm{~mm}$ high swarming. Maximum diameter of swarming zone was $85 \mathrm{~mm}$; minimum diameter of swarming zone was estimated at $10 \mathrm{~mm}$

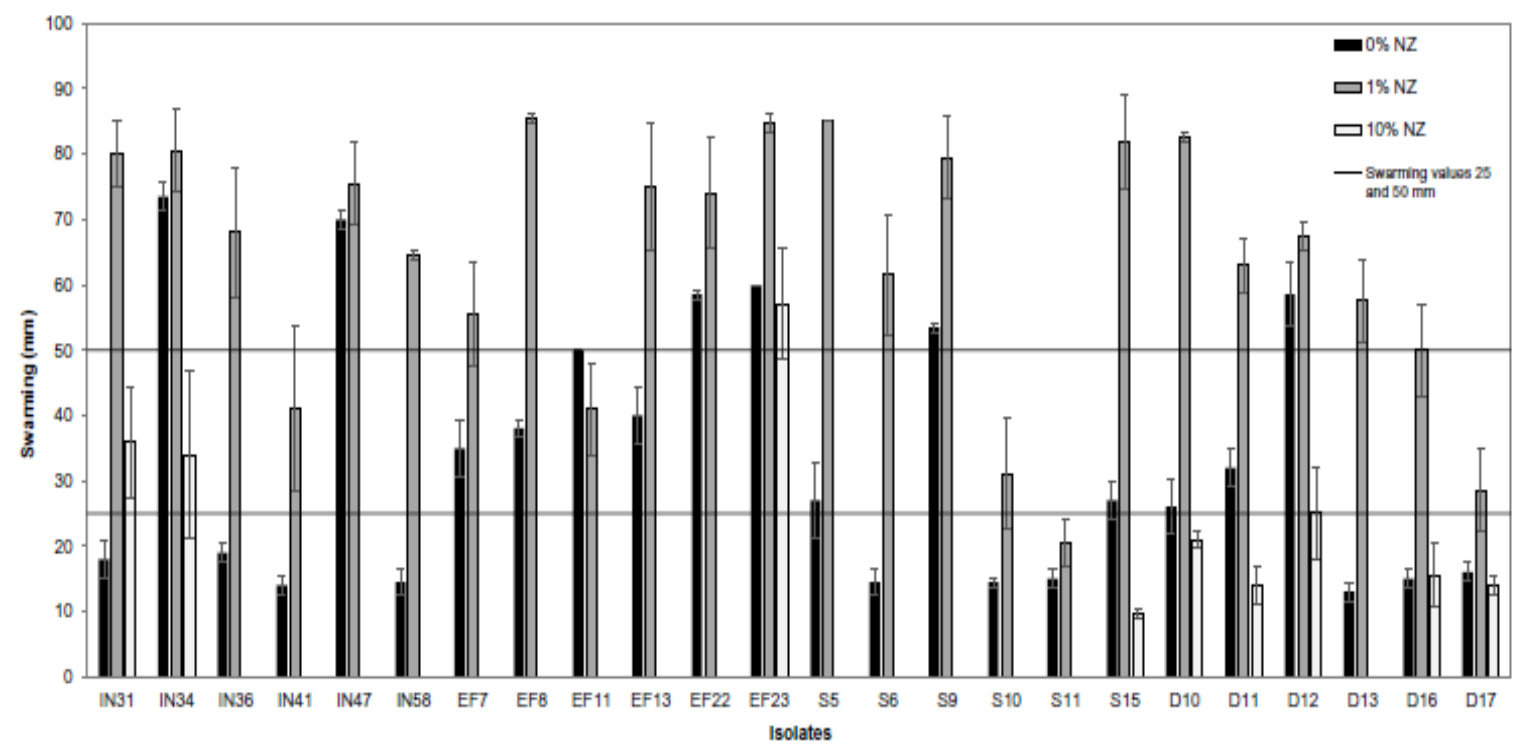

Fig.3 Twitching motility without natural zeolite ( $0 \%$ NZ), with $1 \%$ NZ, and for selected isolates with $10 \%$ of NZ. Lines represent boundaries: <25mmpoor, $25-50 \mathrm{~mm}$ intermediate, $>50 \mathrm{~mm}$ high twitching. Maximum diameter of twitching zone was $85 \mathrm{~mm}$; minimum diameter of twitching zone was $0 \mathrm{~mm}$

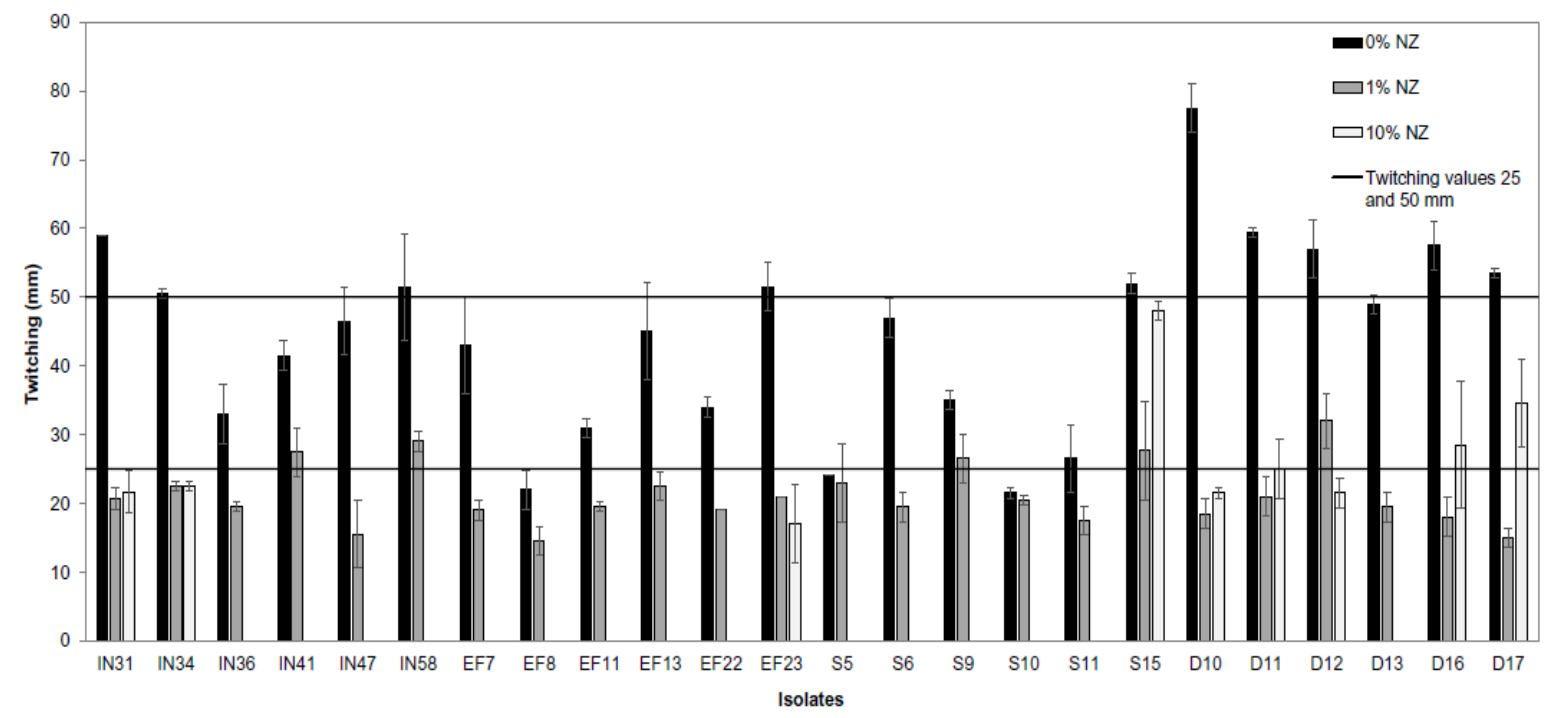


Fig. 4 Cells of Acinetobacter baumannii immobilized onto NZ particles.

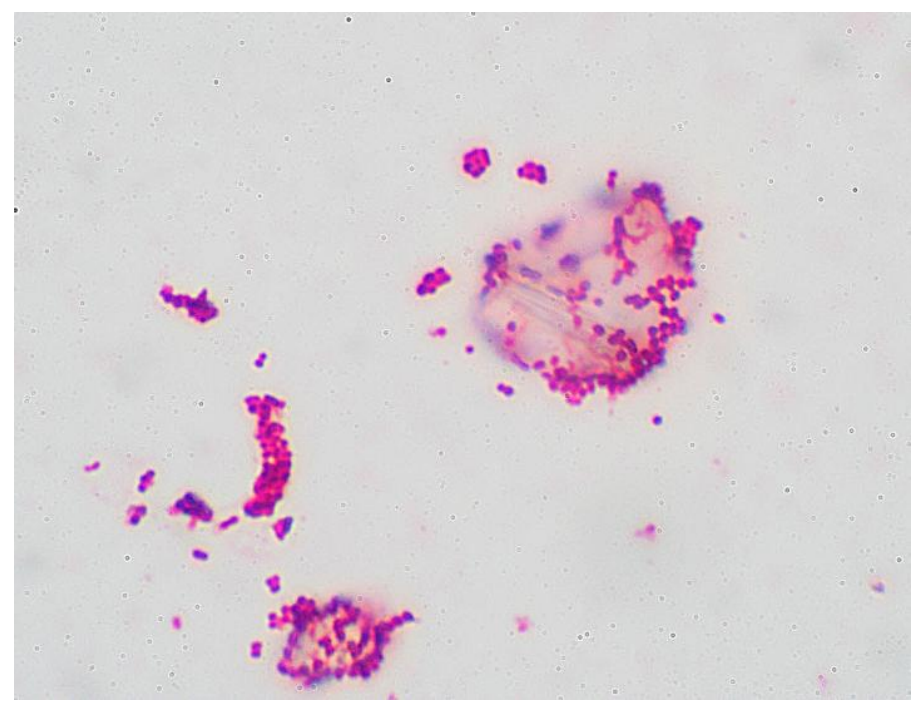

The 24 isolates of A. baumannii recovered from different stages of secondary type municipal wastewater treatment, both antibiotics-sensitive and MDR, showed different expression of the virulence factors that may contribute to its pathogenesis and survival in the natural environment. Ability of the expression of biofilm and pellicle formation, swarming and twitching motility was comparable to those of clinical isolates described in many literature reports(Antunes et al., 2011; Eijkelkamp et al., 2011a; Espinal et al., 2012; Marti et al., 2011; Nait Chabane et al., 2014).

The $38 \%(9 / 24)$ of all isolates showed marked hydrophobicity in BATH assay, suggesting the wastewater as more suitable ecological niche for hydrophilic isolates. Statistically significantly higher hydrophobicity of antibiotics-sensitive isolates as compared to MDR isolates indicates the cell hydrophobicity as a possible protection mechanism against different emerging chemical compounds present in wastewater. Hydrophobicity of cells was statistically significantly positively correlated with biofilm formation at solid-liquid and air- liquid interfaces. Clinical strains of $A$. baumannii that were more hydrophobic also formed stronger biofilm (Kempf et al., 2012) and pellicles (Nait Chabane et al., 2014).Obviously more hydrophobic bacteria form stronger biofilms in order to protect themselves from aqueous medium.

Biofilm formation at solid-liquid and airliquid interfaces of environmental isolates of A. baumannii was significantly positively correlated and mutually linked parameters. Statistically stronger biofilm formation at solid-liquid and air-liquid interfaces was confirmed for antibiotics-sensitive isolates as compared to MDR isolates. This observation is in accordance with statements published for clinical isolates of $A$. baumannii that isolates sensitive to antibiotics form stronger biofilm (Kaliterna et al., 2015; Perez et al., 2015; Qi et al., 2016). Biofilm protects sensitive isolates from the harmful effect of antibiotics, while MDR isolates have already developed mechanisms to protect themselves from antibiotics and therefore do not tend to assemble cells in biofilm. The majority of the examined environmental isolates showed intermediate or high swarming (14/24or 58\% 
of isolates) and twitching (21/24 or $88 \%$ of isolates) motility. Swarming and twitching motility were confirmed to be independent phenotypic parameters. Twitching motility was found to be a common trait of clinical strains of $A$. baumannii which are high biofilm formers (Eijkelkamp et al., 2011a). In this study no correlation of twitching motility and biofilm formation was detected. Twitching motility and biofilm formation on solid surface were shown to depend on the source of clinical isolates of A. baumannii, where blood isolates were more motile and sputum isolates formed stronger biofilm (Vijayakumar et al., 2016). Due to the absence of any significant correlation with the examined parameters, it seems that the surface motility of environmental isolates of A. baumanniiis strain dependant.

Biofilm formation and twitching motility on solid surfaces of environmental isolates of $A$. baumannii were significantly reduced by the addition of $1 \%$ of $\mathrm{NZ}$ into the growth medium, and addition of $10 \%$ of NZ resulted in further suppression of these parameters. Biofilm formation and twitching motility could be reduced up to 85 and $76 \%$, respectively by the addition of only $1 \%$ of NZ. Although the addition of $10 \%$ of NZ did not significantly increase the reduction of twitching motility, it reduced the biofilm formation up to $96 \%$. The effect of NZ addition was evident in experiments on the biofilm formation and twitching motility on solid surfaces where bacteria were in direct contact with the NZ particles. In experiments on the pellicle formation and swarming motility, NZ particles were located at the bottom of the tube or Petri dish and were not in direct contact with bacteria. This explains the lower efficiency of NZ addition on the reduction of pellicle formation and swarming motility.

The reduction of biofilm formation and twitching motility is explained by the immobilization of $A$. baumannii onto $\mathrm{NZ}$ particles. The clinoptilolite content of the NZ used in this study was relatively low (50$55 \%$ ). However, the clinoptilolite content in $\mathrm{NZ}$ was proved not to be the prevailing factor for the immobilization of bacteria (Hrenovic et al., 2009). Another species of the genus Acinetobacter, A. junii, was immobilized in high numbers $\left(1.27 \times 10^{10} \mathrm{CFU} / \mathrm{g}\right)$ onto same NZ of particle size $0.122-0.263 \mathrm{~mm}$ (Hrenovic et al., 2011). The extent of the immobilization onto NZ of particle size $<0.122$ mmwould surely be greater, since the number of immobilized bacteria increase with decrease of particle size (Hrenovic et al., 2005). Immobilization of $A$. baumannii onto NZ particles in this study was not quantified, but is confirmed microscopically. Therefore, the reduction of biofilm formation and twitching motility is explained by the immobilization of A. baumannii onto NZ particles. Obviously, cells of $A$. baumannii were rather attached onto NZ particles than to the surface of polypropylene and polystyrene surfaces of tubes and Petri dish. The affinity of bacteria for NZ could be explained by the rough surface of NZ particles compared to the smooth plastic surfaces. The proportion of bacteria captured by NZ resulted in lower bacterial abundance as compared to the medium without NZ, therefore bacteria were not available for biofilm formation and twitching motility.

Inhibition of expression of the virulence factors of $A$. baumannii by $\mathrm{NZ}$ is promising in control of this emerging human pathogen. NZ particles could find the application in cleaning products, where A. baumannii could be captured by NZ and then easily removed from the contaminated environment.

In conclusion environmental isolates of $A$. baumannii express the virulence factors comparable to the clinical isolates. Isolates sensitive to antibiotics form stronger biofilm 
and pellicles than MDR isolates. Cell surface hydrophobicity is an important feature which determines biofilm and pellicle formation, while swarming and twitching motility seem to be strain dependant. The addition of $1 \%$ of NZ into the growth medium effectively reduced the twitching motility and biofilm formation due to the immobilization of bacterial cells onto NZ particles, while pellicle formation and swarming motility were inhibited only by the addition of $10 \%$ of NZ. NZ is a promising material for the reduction of $A$. baumannii virulence factors and could find application in control of the adherence and subsequent biofilm formation of this emerging pathogen on abiotic surfaces.

\section{Acknowledgement}

This work has been supported by the Croatian Science Foundation (project no. IP-2014-095656). We thank to the staff of Zagreb Wastewater - Management and Operation Ltd. for providing the wastewater and sludge samples.

\section{References}

Antunes, L.C.S., Imperi, F., Carattoli A., Visca, P. 2011. Deciphering the multifactorial nature of Acinetobacter baumannii pathogenicity. PLoS ONE, 6: e22674.

Clinical and Laboratory Standards Institute. 2013. Performance standards for antimicrobial susceptibility testing; Twenty-third informational supplement. CLSI Document M100-S23.

Eijkelkamp, B.A., Hassan, K.A., Paulsen, I.T., Brown, M.H. 2011b. Investigation of the human pathogen Acinetobacter baumannii under iron limiting conditions. $B M C$ Genomics, 12: 1.

Eijkelkamp, B.A., Stroeher, U.H., Hassan, K.A., Papadimitrious, M.S., Paulsen, I.T., Brown,M.H. 2011a. Adherence and motility characteristics of clinical Acinetobacter baumannii isolates. FEMS Microbiol. Lett., 323: 44-51.
Espinal, P., Marti S., Vila J. 2012. Effect of biofilm formation on the survival of Acinetobacter baumannii on dry surfaces. $J$. Hosp. Infect. ,80: 56-60.

European Committee on Antimicrobial Susceptibility Testing. 2016. EUCAST Reading guide. Version6.0. Vaxjo: EUCAST.

Ferreira, A.E., Marchetti, D.P., De Oliveira, L.M.,Gusatti, C.S., $\quad$ Fuentefria, D.B.,Corção, G. 2011. Presence of OXA23-producing isolates of Acinetobacter baumannii in wastewater from hospitals in southern Brazil. Microb. Drug Resist., 17: 221-227.

Girlich, D., Poirel, L., Nordmann, P. 2010. First isolation of the blaOXA-23 carbapenemase gene from an environmental Acinetobacter baumannii isolate. Antimicrob. Agents Chemother., 54: 578-579.

Goic-Barisic,I., Seruga Music, M., Kovacic,A., Tonkic, M., Hrenovic,J. 2017. Pan drugresistant environmental isolate of Acinetobacter baumannii from Croatia. Microb. Drug Resist., DOI:10.1089/mdr.2016.0229

Hrenovic, J., Durn, G., Goic-Barisic, I., Kovacic, A. 2014. Occurrence of an environmental Acinetobacter baumannii strain similar to a clinical isolate in paleosol from Croatia. Appl. Environ. Microbiol., 80: 2860-2866.

Hrenovic, J., Goic-Barisic, I.,Kazazic, S., Kovacic, A., Ganjto, M., Tonkic,M. 2016. Carbapenem-resistant isolates of Acinetobacter baumannii in a municipal wastewater treatment plant, Croatia, 2014. Euro Surveill. 21: 21-30.

Hrenovic, J., Ivankovic, T., Tibljas, D. 2009. The effect of mineral carrier composition on phosphate-accumulating bacteria immobilization. J. Hazard. Mater., 166: 1377-1382.

Hrenovic, J., Kovacevic, D., Ivankovic, T., Tibljas,D. 2011. Selective immobilization of Acinetobacter junii on the natural zeolitized tuff in municipal wastewater. Colloids Surf. B: Biointerfaces, 88: 208214.

Hrenovic, J., Tibljas, D., Orhan, Y., Büyükgüngör, H. 2005. Immobilisation of Acinetobacter calcoaceticus using natural 
carriers. Water SA, 31: 261-266.

Kaliterna, V., Kaliterna, M., Hrenovic, J., Barisic, Z., Tonkic, M., Goic-Barisic, I. 2015. Acinetobacter baumannii in the Southern Croatia: clonal lineages, biofilm formation and resistance patterns. Infect. Dis., 47: 902-907.

Kempf, M., Eveillard, M., Deshayes, C., Ghamrawi, S., Lefrançois, C., Georgeault, S., Bastiat, G., Seifert, H., Joly-Guillou, M.L. 2012. Cell surface properties of two differently virulent strains of Acinetobacter baumannii isolated from a patient. Can. J. Microbiol., 58: 311-317.

Marti, S., Rodríguez-Baño, J., Catel-Ferreira, M., Jouenne, T., Vila, J., Seifert,H., Dé, E. 2011. Biofilm formation at the solid-liquid and air-liquid interfaces by Acinetobacter species. BMC Res. Notes, 4: 5.

McConnell, M.J., Actis,L., PachónJ.2013. Acinetobacter baumannii: human infections, factors contributing to pathogenesis and animal models. FEMS Microbiol. Rev., 37: 130-155.

Mussi, M., Gaddy, J.A., Cabruja, M., Arivett, B.A., Viale, A.M., Rasia, R., Actis,L.A. 2010. The opportunistic human pathogen Acinetobacter baumannii senses and responds to light. J. Bacteriol., 192: 63366345.

Nait Chabane, Y., Marti, S., Rihouey, C., Alexandre, S., Hardouin, J., Lesouhaitier, O., Vila, J., Kaplan, J.B., Jouenne, T., Dé, E. 2014. Characterisation of pellicles formed by Acinetobacter baumannii at the air-liquid interface. PLoS ONE, 9: e111660.

Perez, L.R. 2015. Acinetobacter baumannii displays inverse relationship between meropenem resistance and biofilm production. J. Chemother., 27: 13-16.

Qi, L., Li, H., Zhang, C.,Liang, B., Li, J., Wang, L., Du, X., Liu, X., Qiu, S., Song, H. 2016. Relationship between antibiotic resistance, biofilm formation, and biofilm-specific resistance in Acinetobacter baumannii. Front. Microbiol., 7: 483.

Rosenberg, M., Gutnick, D., Rosenberg, E. 1980. Adherence of bacteria to hydrocarbons: a simple method for measuring cell-surface hydrophobicity. FEMS Microbiol. Lett., 9: 29-33.

Sousa, C., Botelho, J., Silva, L., Grosso, F., Nemec, A., Lopes, J., Peixe, L. 2014. MALDI-TOF MS and chemometric based identification of the Acinetobacter calcoaceticus-Acinetobacter baumannii complex species. Int. J. Med. Microbiol., 304: 669-677.

Towner, K.J. 2009. Acinetobacter: an old friend, but a new enemy. J. Hosp. Infect., 73: 355363.

Vijayakumar, S., Rajenderan, S., Laishram, S.,Anandan, S., Balaji, V.,Biswas, I.2016. Biofilm formation and motility depend on the nature of the Acinetobacter baumannii clinical isolates. Front. Public. Health, 4: 105 .

Wong T.W.2009. Handbook of Zeolites: Structure, Properties and Applications. New York (NY), Nova Science Publishers Inc.

Zhang, C., Qiu, S., Wang, Y., Qi, L., Hao, R., Liu, X., Shi, Y., Hu, X., An, D., Li, Z., Li, P., Wang, L., Cui, J., Wang, P., Huang, L., Klena, J.D., Song, H. 2013. Higher isolation of NDM-1 producing Acinetobacter baumannii from the sewage of the hospitals in Beijing. PLoS ONE, 8: e64857.

\section{How to cite this article:}

Svjetlana Dekic, Jasna Hrenovic, Blazenka Hunjak, Snjezana Kazazic, Darko Tibljas and Tomislav Ivankovic. 2017. Virulence Factors of Acinetobacter baumannii Environmental Isolates and Their Inhibition by Natural Zeolite. Int.J.Curr.Microbiol.App.Sci. 6(3): 1697-1709. doi: https://doi.org/10.20546/ijcmas.2017.603.195 